\title{
Unified Model for Dark Energy
}

\author{
Pedro F. González-Díaz \\ Centro de Física "Miguel A. Catalán", Instituto de Matemáticas y Física Fundamental, \\ Consejo Superior de Investigaciones Cientificas, Serrano 121, 28006 Madrid (SPAIN).
}

(Dated: November 2, 2018)

\begin{abstract}
A new model for the universe filled with a generalized Chaplygin fluid is considered which unitarily describes as a single vacuum entity both a quintessence scalar field and a cosmological constant, so unifying the notion of dark energy. While the evolution of the universe filled with such a fluid does not obviously contradict the present cosmic acceleration, the introduced single dark-energy component, for equations of state with characteristic parameter $\omega \geq-1$, behaves like an usual quintessence fluid with constant equation of state at early high densities, and like a pure cosmological constant at late cosmological times.
\end{abstract}

PACS numbers: 98.80.-k, 98.80.Es

Rather surprising new evidence for dark energy from a ten-year census of the gravitational lensing in distant quasars has been quite recently added by Browne et al. [1] to the itself compelling evidence originally obtained from distant supernova $[2,3]$ and all those ever favorable results later amassed in the past five years [4]. Thus, it now appears as a rather solid conclusion that the present universe is accelerating and that most of its energy is in the form of a mysterious dark energy, which has longrange anti-gravity properties. Dark energy is closely related to the idea of a positive cosmological constant, or to the notion of the so-called cosmic quintessence [5], a slowly-varying, tracked [6] or not [7], scalar field that produces a negative pressure. But, whatever its ultimate nature, dark energy has become one of the main problems of all sciences [8].

On the other hand, dark energy has also been claimed [9] to be just one of the two vacuum-energy components in a unified model for cosmic darkness whose other component is dark matter. Rather than fine-tuning any specific quintessence potential, this model explains the present accelerating expansion of the universe by means of the rather exotic equation of state that corresponds to a generalized Chaplygin gas [10], acting in such a way that it caused the gas to behave like pure dark matter at early times (high density) and like dark energy at late times (small density). The generalized Chaplygin gas is a substance which is characterized by an equation of state [9-11]

$$
p=-A \rho^{-\alpha},
$$

with $A$ a positive definite constant and $\alpha$ a parameter which can take on positive or negative values. The original Chaplygin gas was characterized by $\alpha=1$. It is an equation of state with a form as given by Eq. (1) that has thus been claimed to represent the stiff that simultaneously describes dark matter and dark energy. In this letter we generalize the idea of Chaplygin gas still one step further in such a way that, rather than unitarily characterizing dark matter and dark energy as a single entity, the resulting gas theory would now simultaneously describe the physics of a quintessence scalar field and that of a cosmological constant as the two opposite limiting situations that result at early and late times, respectively, from a unique unified dark energy physical picture.

The dynamical cosmological equations that represent a pure quintessence field with constant state equation [7] $p=\omega \rho$ are known to be valid only in an $\omega$-interval, $0 \leq \omega<-1$, which cannot include the value $\omega=-1$ that corresponds to a pure cosmological constant $\Lambda$ [12]. The generalization of the Chaplygin gas that we are going to introduce does neither require specifying any potential for the generalized field and is based on suitably combining the state equations for a slowly-varying quintessence scalar field with that for the generalized Chaplygin gas given by Eq. (1), in such a way that the resulting state equation would give rise to cosmological dynamical equations that would represent both a quintessence-like field and a cosmological constant in the above single unified way. We take for that state equation the following simplest combination

$$
p=\left(\omega-\frac{A}{\rho^{\alpha+1}}\right) \rho,
$$

where $p$ and $\rho$ are respectively pressure and energy density in a comoving reference frame, with $p<0$ and $\rho>0,0 \geq \omega>-1$ and $A$ is again a constant, but one which should not be necessarily positive definite, provided $p \leq 0$. This equation of state is interesting as it can be connected with both cosmology through parameter $\omega$ and string theory through its Chaplygin-like gas component [9]. Inserting Eq. (2) into the general cosmic conservation law for energy [13]

$$
d \rho=-3(\rho+p) \frac{d a}{a},
$$

we obtain for the density of the dark energy Chaplygin gas a nonlinear expression in terms of the scale factor $a$. It is:

$$
\rho(a)=\left\{\frac{1}{1+\omega}\left[A+\frac{B}{a^{3(1+\alpha)(1+\omega)}}\right]\right\}^{1 /(1+\alpha)},
$$

with $B$ an integration constant and $a \equiv a(t)$ the cosmic scale factor which is normalized to unity today 
$a=(1+z)^{-1}$, when expressed in terms of the redshif $z$. A differential feature of the present model in relation with the unified dark matter model is that, although the density never drop below the value $A^{1 /(1+\alpha)}$, no matter how much you expand the gas, it get a value $\rho \propto a^{-3(1+\omega)}$ when sufficiently compressed, so that the density can never reach the value $\rho \propto a^{-3}$ if $\omega<0$.

Let us now introduce the re-definitions for the current generalized dark-energy field $\phi$ cosmological parameter

$$
\Omega_{\phi}^{0}=\frac{B}{A+B} .
$$

and energy density

$$
\rho_{0}=\left[\frac{A+B}{1+\omega}\right]^{1 /(1+\alpha)} .
$$

In terms of these quantities Eq. (4) can be re-written in a form which is similar to that has been used in unified dark matter models [14]

$$
\rho(a)=\rho_{0}\left[1-\Omega_{\phi}^{0}+\frac{\Omega_{\phi}^{0}}{a^{3(1+\omega)(1+\alpha)}}\right]^{1 /(1+\alpha)} .
$$

In order to interpret this equation, let us consider the corresponding expression for the energy density in the case that we have a quintessence slowly-varying scalar field and a cosmological constant which are separatedly defined in the flat cosmological case [15], i.e.

$$
\rho(a)=\rho_{0}\left[\left(1-\Omega_{\Lambda}\right) a^{-3\left(1+\omega_{0}\right)}+\Omega_{\Lambda}\right] .
$$

We first note that, although Eq. (5) involves a single substance and Eq. (6) involves two, the energy densities given by these two equations become both the density $\rho_{0}$ for the current value $(z=0)$ of the scale factor $a=$ $1 /(1+z)=1$. Making then the identifications

$$
\Omega_{\phi}^{0}=\left(1-\Omega_{\Lambda}\right)^{1+\alpha}, \quad \omega=\omega_{0},
$$

we see that Eqs. (5) and (6) again become the same and are both given by

$$
\rho=\rho_{0} \Omega_{\phi}^{0} a^{-3(1+\omega)}
$$

in the limit $a \rightarrow 0$. It follows that $\Omega_{\phi}$ behaves like though it were an effective quintessence density in the considered model. Also for the flat case, once again Eqs. (5) and (6) become the same when $\alpha=0$ and are both given by

$$
\rho=\rho_{0}\left[\Omega_{\phi}^{0}+\Omega_{\Lambda} a^{-3(1+\omega)}\right]
$$

which corresponds to the standard combined " $\Lambda$ Quintessence" model which involves two substances. Finally, Eqs. (5) and (6) become also the same in the limit $a \rightarrow \infty$, where both read

$$
\rho=\rho_{0} \Omega_{\Lambda}
$$

in case that the identification $\Omega_{\Lambda}=\left(1-\Omega_{\phi}^{0}\right)^{1 /(1+\alpha)}$ be also introduced. This latter case would clearly correspond to that for a pure cosmological constant.

The interpretation of our present picture for dark energy which describes quintessence and cosmological constant in a unified, single fashion can be further strengthened if we also introduce definitions for the effective parameter entering the equation of state and the effective speed of sound, which in the present case become

$$
\omega^{\mathrm{eff}}=\frac{p}{\rho}=\omega-\frac{(1+\omega) A}{A+\frac{B}{a^{3(1+\omega)(1+\alpha)}}}
$$

$$
c_{s}^{\mathrm{eff} 2}=\frac{\partial p}{\partial \rho}=\omega+\frac{(1+\omega) \alpha A}{A+\frac{B}{a^{3(1+\omega)(1+\alpha)}}}
$$

We notice that both $\omega^{\text {eff }}$ and $c_{s}^{\text {eff2 }}$ reduce to $\omega$ (the typical pure quintessential case) when $a \rightarrow 0$, and to $\omega^{\text {eff }}=-1$, $c_{s}^{\text {eff2 }}=\alpha+\omega(1+\alpha)$ (the cosmological constant case) whenever $a \rightarrow \infty$. This closes up the proof that our model consistently describes both quintessence and cosmological constant as given limiting concepts from a more general unified dark energy description involving one single substance, and can therefore be looked at as being a promising concept for cosmology.

However, a wide class of the so-called unified dark energy models, which are similarly constructed using a less generalized Chaplygin equation of state, has been recently excluded [14] because such models give rise to oscillations or exponential browup in the matter power spectrum that are inconsistent with observations. One would in fact expect these unwanted phenomena to occur as well in our present unified dark energy model. Following Sandvik et al. [14], the evolution of density perturbations $\delta_{k}$ with wave vector $k$ can be written in the form [16]:

$$
\begin{aligned}
& \delta_{k}^{\prime \prime}+\left[2+\xi-3\left(2 \omega^{\mathrm{eff}}-c_{s}^{\mathrm{eff} 2}\right)\right] \delta_{k}^{\prime} \\
& -\left[\frac{3}{2}\left(1-6 c_{s}^{\mathrm{eff} 2}+8 \omega^{\mathrm{eff}}-3 \omega^{\mathrm{eff} 2}\right)\right. \\
& \left.-\left(\frac{k c_{s}^{\mathrm{eff}}}{a H}\right)^{2}\right] \delta_{k}=0
\end{aligned}
$$

where $H$ is the Hubble parameter,

$$
\xi=-\frac{2}{3}\left[1+\left(\frac{1}{\Omega_{\phi}^{0}}-1\right) a^{3(1+\alpha)}\right]^{-1},
$$

and the prime denotes differentiation with respect to the timelike independent variable $\ln a$. By inspecting Eq. (9) it can in fact be inferred that whenever the effective speed of sound is nonzero, the unwanted oscillatory $\left(c_{s}^{\text {eff }}>0\right)$ and exponential blowup $\left(c_{s}^{\text {eff }}<0\right)$ behaviours of fluctuations with wavelengths below the Jeans scale $\sqrt{\pi\left|c_{s}^{\mathrm{eff}}\right|^{2} / G \rho}$ would always take place. Whereas this situation is unavoidable for the wide class of unified dark 
matter models pointed out by Sandvik et al. [14], here one always has the possibility to set $c_{s}^{\text {eff }}=0$ at all times, while keeping a nonzero value for the bare speed of sound,

$$
c_{s}^{2}=\frac{A \alpha}{A+\frac{B}{a^{3(1+\omega)(1+\alpha)}},}
$$

because the constant $A$ may in our case take also on negative values while the right-hand-side of Eq. (8) has two terms to play with. Thus, restricting to the simplest case where we choose $\alpha=-1$, and hence $\omega^{\text {eff }}=0$, the fluctuations appear to be free from the unwanted oscillatory and exponential blowup behaviours. In this case, one has to take negative values for the cosmological constant $A$ [a case which can have very positive implications (see later on)], so that Eq. (9) reduces to:

$$
\delta^{\prime \prime}+\left(2-\frac{3}{2} \Omega_{\phi}^{0}\right) \delta^{\prime}-\frac{3}{2} \delta=0 .
$$

The solutions to Eq. (10) are

$$
\delta_{ \pm}=a^{\frac{3}{4} \Omega_{\phi}^{0}-1 \pm \sqrt{1+\frac{9}{16}\left(\Omega_{\phi}^{0}\right)^{2}-\frac{3}{2}\left(\Omega_{\phi}^{0}-1\right)}}
$$

at all times. We have thus shown that the unstability problem and the presence of unobserved oscillations affecting most of the unified dark matter models can both be avoided in the kind of models considered here for unified dark energy.

All the above kinds of models are however unrealistic, unless as asymptotic approximations, because they describe an asymptotic universe devoid of observable matter. One therefore ought to include some kind of observable matter and/or radiation contents in order to make the models more realistic. This will be later implemented by considering a FRW scenario where, besides the unified dark energy field, a radiation field will also be included. We shall classically describe such a radiation field as a massless scalar field which is conformally coupled to gravity $[15,17]$. Before doing that, we shall first consider the simplest case of a spatially flat universe filled only with the single generalized Chaplygin gas discussed in this letter that satisfies the state equation given by Eq. (2). The Friedmann equation for this problem is

$$
\left(\frac{\dot{a}}{a}\right)^{2(1+\alpha)}=\frac{1}{1+\omega}\left[A+\frac{B}{a^{3(1+\alpha)(1+\omega)}}\right],
$$

where the overhead dot means time derivative. As pointed out above, the solution to Eq. (11) for the general case in which $\alpha \neq 0$ can only match the solution to the Friedmann equation for the simple case that corresponds to a quintessence field with constant equation of state $p=\omega \rho$ plus a cosmological constant $\Lambda=3 H^{2} \lambda$, that is for e.g. an allowed value of the initial, constant parameter $\omega$ whose following evolution be able to reproduce the current cosmic acceleration, $\omega=-2 / 3$,

$$
a(t)=\frac{8 \pi G}{3 \sigma \lambda} \sinh ^{2}\left(\frac{1}{2} \sqrt{3 \lambda} t\right),
$$

just in the limiting cases of small $a$ as $\alpha \rightarrow 0$, with $A=\lambda$ and $B=8 \pi G /(3 \sigma)$, in which $\sigma$ is another integration constant. Note that for negative cosmological constant the above solution changes into

$$
a(t)=\frac{8 \pi G}{3 \sigma|\lambda|} \sin ^{2}\left(\frac{1}{2} \sqrt{3|\lambda|} t\right) .
$$

For the small values of $|\lambda|$ and $a$ for which this oscillating solution is valid, it would be expected that the universe will show [18] no future event horizon, a property which has been claimed [19] to be of decisive importance for allowing a consistent mathematical formulation of fundamental string and $\mathrm{M}$ theories.

Applying the generalization considered in this letter to the original Chaplygin gas $(\alpha=1)$, one obtains from Eq. (11) for the flat case in the simple situation that corresponds to the initial equation of state parameter $\omega=-2 / 3$ (which is compatible with the present cosmic accelerating expansion for moderate values of $A /(A+B)$ and the most recent observational constraints $[20])$,

$$
\dot{a}^{4}=3\left(A a^{4}+B a^{2}\right) .
$$

A closed-form general analytical solution to Eq. (14) can be obtained for the scale factor $a$ in parametric form and is given by

$$
t=(3 A)^{-1 / 4}\left\{\ln \left[\frac{1+F(a)}{1-F(a)}\right]^{1 / 2}-\arctan [F(a)]\right\}+\zeta,
$$

where $\zeta$ is an adjustable integration constant and

$$
F(a)=\left(1+\frac{B}{A a^{2}}\right)^{1 / 4}=\left(1+\frac{\Omega_{\phi}^{0}}{\Omega_{\Lambda}^{0} a^{2}}\right)^{1 / 4} .
$$

Choosing

$$
\zeta=\frac{(1-i) \pi}{2(3 A)^{1 / 4}}
$$

for the integration constant so that $a \rightarrow 0$ as $t \rightarrow 0$, we finally get

$$
t=(3 A)^{-1 / 4}\left\{\ln \left[\frac{F(a)+1}{F(a)-1}\right]^{1 / 2}+\frac{\pi}{2}-\arctan [F(a)]\right\}
$$

It follows from this solution that as the single gas is evolving from a pure quintessence field into a cosmological term, the scale factor $a$ will increses from zero, at $t=0$, to infinity, as $t \rightarrow \infty$, such as one should expect in the unified picture for dark energy.

Let us next include a term in the Friedmann equation that accounts for the existence of an observable radiation field. As we mentioned before, we represent this field by means of a massless scalar field which is conformally coupled to gravity [17] so that one has to modify [21] the Friedmann equation (11) to read:

$$
\left[\left(\frac{\dot{a}}{a}\right)^{2}-\frac{M^{2}}{a^{4}}\right]^{1+\alpha}=\frac{1}{1+\omega}\left[A+\frac{B}{a^{3(1+\alpha)(1+\omega)}}\right],
$$


where $M^{2}$ is an integration constant. Although we have been unable to find any analytical solution to Eq. (17) in closed-form, it is still possible to readily deduce that in the limit of very small values of the scale factor we have the approximate solution

$$
a(t)=\sqrt{2 M t},
$$

for the entire range of allowed values of parameter $\omega \leq 0$. It follows that radiation dominated over the unified dark energy at early time. At late time the unified dark energy field would start dominating over radiation, as one would expect, so that it will be described by Eq. (16) (or any of its counterpart solutions for $\alpha \neq 1$ ) which would govern the evolution of the current universe. It appears therefore that during the time along which the generalized Chaplygin dark-energy gas behaved like a quintessence field at the earliest times, this was nevertheless dominated by radiation; however, at the late times when that generalized single gas behaves like a cosmological constant, this dominated over radiation. We therefore hope that a more detailed consideration of unified dark energy may lead us to solve, or at least greatly alleviate the cosmic coinci- dence problem [22]. Before closing up, we mention the interesting possibility that the unified dark energy can also behave like a quintessence field when $a \rightarrow \infty$ and like a cosmological constant as $a \rightarrow 0$. That would happen if $\omega<-1$, with $A$ and $B$ taking on negative values (so keeping the current density, the quintessence energy density and the cosmological constant all positive), a case which is not excluded by current constraints on the state equation for dark energy [20]. We finally note that Eq. (2) would reduce to Eq. (1) in case that $\alpha<0$ as $a \rightarrow 0$, so indicating that in such a case our unified model for dark energy also included the current unified model for dark matter in the limit of high density.

\section{Acknowledgments}

The author thanks Mariam Bouhmadi and Carmen L. Sigüenza for useful conversations. This work was supported by MCYT under Research Project No. BMF200203758 .
[1] I.W.A. Browne et al., astro-ph/0211069

[2] S. Perlmutter et al., Astrophys. J. 483, 1565 (1997).

[3] A.G. Riess et al., Astron. J. 116, 1009 (1998).

[4] P. de Bernardis et al., Nature 404, 955 (2000); T. Moroi and H. Murayama, hep-ph/0211019

[5] C. Wetterich, Nucl. Phys. B302, 668 (1988); B. Ratra and P.J.E. Peebles, Phys. Rev. D37, 3406 (1988); J.A. Frieman, C.T. Hill, A. Stebbins and I. Waga, Phys. Rev. Lett. 75, 2077 (1995); P.G. Ferreira and M. Joyce, Phys. Rev. Lett. 79, 4740 (1997); V. Sahni and A.A. Starobinsky, Int. J. Mod. Phys. A9, 373 (2000); S.M. Carroll, Living Review in Relativity 4, 1 (2001).

[6] I. Zlatev, L. Wang and P.J. Steinhardt, Phys. Rev. Lett. 82, 896 (1999); P.J. Steinhardt, L. Wang and I. Zlatev, Phys. Rev. D59, 123504 (1999); I. Zlatev and P.J. Steinhardt, Phys. Lett. B459, 570 (1999); P. Brax, J. Martin and A. Riazuelo, Phys. Rev. D62, 103505 (2000).

[7] R.R. Caldwell, R. Dave and P.J. Steinhardt, Phys, Rev. Lett. 80, 1582 (1998); L. Wang and P.J. Steinhardt, Astrophys. J. 508, 483 (1998); R.R. Caldwell and P.J. Steinhardt, Phys. Rev. D57, 6057 (1998); P.F. González-Díaz, Phys. Rev. D62, 023513 (2000).

[8] M.S. Turner, astro-ph/0207297

[9] A. Kamenshchik, U. Moschella, V. Pasquier, Phys. Lett. B511, 265 (2001); N. Bilic, G.B. Tupper and R. Viollier, Phys. Lett. B535, 17 (2001); M.C. Bento, O. Bertolami and A.A. Sen, astro-ph/0202064 J.S. Fabris, S.V. Gonzalves and P.E. de Souza, Gen. Rel. Grav. 34, 53 (2002); V. Gorini, A. Kamenshchik and U. Moschella, astro-ph/0209395 A. Dev., D. Jain and J.E. Alcaniz, astro-ph/0209379

[10] J.D. Barrow, Phys. Lett. B180, 335 (1986); B235, 40 (1990); D. Bazcia and J. Jackiw, Ann. Phys. 270, 246 (1998).
[11] M. Makler, S. Quinet de Oliveira and I. Waga, astro-ph/0209486

[12] E. di Pietro and J. Demaret, Int. J. Mod. Phys. D10, 231 (2001).

[13] E.W. Kolb amd M.S. Turner, The Early Universe (Addison-Wesley, Redwood City, CA, USA, 1990); T. Padmanabhan, Cosmology and Astrophysics (Cambridge University Press, Cambridge, UK, 1996).

[14] D. Carturan and F. Finelli, astro-ph/0211626 H.B. Sandwik, M. Tegmark, M. Zaldarriaga and I. Waga, astro-ph/0212114

[15] P.F. González-Díaz, astro-ph/0211069

[16] T. Padmanabhan, Structure Formation in the Universe (Cambridge University Press, Cambridge, UK, 1993).

[17] J.J. Halliwell and E. Laflamme, Class. Quant. Grav. 6, 1839 (1989).

[18] P.F. González-Díaz, Phys. Lett. B522, 211 (2001); Phys. Rev. D65, 104035 (2002).

[19] W. Fischler, A. Kashani-Poor, R. McNees and S. Paban, JHEP 0107 (2001); S. Hellman, N. Kaloper and L. Susskind, JHEP 0106 (2001); L. Dyson, M. Kleban and L. Susskind, JHEP 0210 (2002).

[20] A. Melchiorri, L. Mersini, C.J. Odman and M. Tradden, astro-ph/0211522 M. Douspis, A. Riazuelo, Y. Zolnierowski and A. Blanchard, astro-ph/0212097

[21] Eq. (17) can be derived from an action for Hilbert-Einstein gravity $S=$ $\int d^{4} x \sqrt{-g}\left[\left(1-4 \pi G \varphi^{2} / 3\right) R+L_{\text {gas }}+L_{\varphi}\right] / 16 \pi G+S$, where $L_{\text {gas }}$ is the Lagrangian for our generalized Chaplygin gas, $L_{\varphi}$ is the Lagrangian for the conformally coupled scalar field $\varphi$, and $S$ represents a boundary integral which does not involve the generalized Chaplygin gas, specializing to a FRW metric $d s^{2}=-d t^{2}+a(t)^{2} d \Omega_{3}^{2}$, with $d \Omega_{3}^{2}$ the metric on the unit three-sphere. 
[22] M. Bastero-Gil and L. Mersini, astro-ph/0205271 